\title{
Joint ASV/AUV Range-Based Formation Control: Theory and Experimental Results
}

\author{
Jorge M. Soares, A. Pedro Aguiar, António M. Pascoal and Alcherio Martinoli
}

\begin{abstract}
The use of groups of autonomous marine vehicles has enormous potential in numerous marine applications, perhaps the most relevant of which is the surveying and exploration of the oceans, still widely unknown and misunderstood. In many mission scenarios requiring the concerted operation of multiple marine vehicles carrying distinct, yet complementary sensor suites, relative positioning and formation control becomes mandatory. However, the constraints placed by the medium make it hard to both communicate and localize vehicles, even in relation to each other. In this paper, we deal with the challenging problem of keeping an autonomous underwater vehicle in a moving triangular formation with respect to 2 leader vehicles. We build upon our previous theoretical work on range-only formation control, which presents simple feedback laws to drive the controlled vehicle to its intended position in the formation using only ranges obtained to the leading vehicles with no knowledge of the formation path. We then introduce the real-world constraints associated with the use of autonomous underwater vehicles, especially the low frequency characteristics of acoustic ranging and its unreliability. We discuss the required changes to implement the solution in our vehicles, and provide simulation results using a full dynamic and communication model. Finally, we present the results of real world trials using MEDUSA-class autonomous marine vehicles.
\end{abstract}

\section{INTRODUCTION}

Recent developments witnessed in the field of autonomous marine vehicles, with increasingly powerful and affordable vehicles coming on the market, open the door for a multitude of novel applications in fields as diverse as biology and defense. Most of the tasks that are envisioned to be within the reach of multi-AUV (Autonomous Underwater Vehicle) groups in the near future require the vehicles to cooperate and localize themselves; other tasks require the vehicles to move in formation, i.e., while keeping their relative positions. Conventional examples include seabed mapping and ocean sampling, and even the ability to use odor tracking algorithms similar to crosswind formation [1] for the detection of underwater plumes emitted by, for instance, a leaking pipeline or a natural hydrothermal vent.

J. M. Soares, A. P. Aguiar and A. M. Pascoal are with the Laboratory of Robotics and Systems in Engineering and Science, Instituto Superior Técnico, Technical University of Lisbon, Av. Rovisco Pais, 1049-001 Lisboa, Portugal.

A. P. Aguiar is with the Department of Electrical and Computer Engineering, Faculty of Engineering, University of Porto, Rua Dr. Roberto Frias, 4200-465 Porto, Portugal.

J. M. Soares and A. Martinoli are with the Distributed Intelligent Systems and Algorithms Laboratory, School of Architecture, Civil and Environmental Engineering, École Polytechnique Fédérale de Lausanne (EPFL), CH-1015 Lausanne, Switzerland.

This work was supported by projects CONAV/FCT-PT [PTDC/EEACRO/113820/2009], MORPH [EU FP7 ICT 288704], and FCT [PEst-OE/EEI/LA0009/2011]. Partially funded with grant SFRH/BD/51073/2010 from Fundação para a Ciência e Tecnologia
In this paper, we consider the problem of triangular formation keeping under severe communication and localization constraints, conditions typically found when working with AUVs. It follows up on our earlier publication on the theoretical design of a simple control approach for range-only triangular formation keeping and presents novel experimental results using real robots in a marine setting. For a reference scenario consisting of 2 localized leader vehicles on the surface and an underwater following vehicle, we use acoustic ranging and communications to establish and maintain a moving formation of the 3 vehicles. Of the multiple realworld applications matching this scenario, a typical one is surface-guided underwater search. We make a realistic assumption that the AUV has independent depth control, and focus on formation control on a 2D plane only.

In the first part of this paper we summarize our previously published range-only approach to formation control using a kinematic model (written in terms of speed and heading) for the follower vehicle [2]. This contribution proposes a control strategy that estimates the formation speed and heading from the ranges obtained to the two leading vehicles, and uses simple feedback laws for speed and heading to drive suitably defined common and differential errors to zero. The original paper includes a proof of local convergence of the distance errors under straight-line motion and specific conditions.

In the second part, we discuss the implementation of this solution in a real vehicle (a MEDUSA-class autonomous marine vehicle), describing the constraints placed by the medium and the alterations required to do so. These include, specifically, estimating the continuous distance signals from discrete samples and replacing heading estimation with piggybacking of the heading on the range replies. Realworld trial results are presented, and compared to simulation results. These are, to our knowledge, the first published realworld results for a range-based formation control strategy for AUVs. While obtained using a surface-bound vehicle, typical constraints affecting an underwater vehicle were observed.

The paper is organized as follows: in the remainder of this section, we present some related work and an overview of the problem at hand; in Section II we review the range-only error dynamics and control laws for linear velocity and heading; in Section III we introduce the necessary adaptations for implementation in a real vehicle; in Section IV we present both simulation and real world testing results. Finally, in Section V, we present our conclusions and future work.

\section{A. Related work}

Related work on formation control can be found in [3], where a leader-follower control problem for a formation 


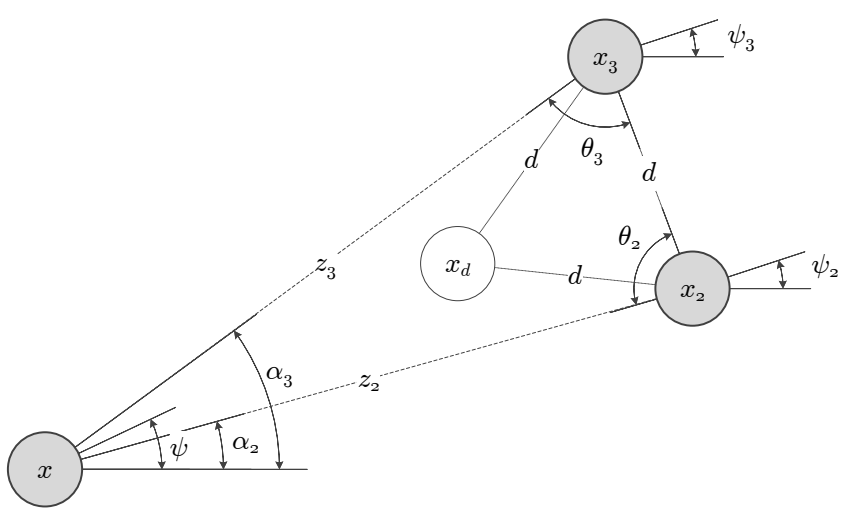

Fig. 1. System of 3 robots $\left(x, x_{2}, x_{3}\right)$ and their intended triangular formation $\left(x_{d}, x_{2}, x_{3}\right)$. The image shows many of the relevant parameters, including the formation and independent vehicle headings, as well as the relationships (ranges and bearings) between them.

graph with an arbitrary number of vehicles is described. The authors discuss approaches for both range-bearing and rangerange control, depending on the available sensors; in both cases, knowledge of the leader motion is assumed. A distinct graph-based leader-follower solution using range and bearing is proposed in [4] and supported by robot experiments.

Another strategy is described in [5], where a solution is proposed for a 4-vehicle station keeping problem, using exclusively range measurements and holonomic vehicles described by simple kinematic points. A similar scenario is considered in [6], although global convergence is only proved for a triangular formation. Bearing-only methods are also available for square [7] and triangular [8], [9] formations.

In [10] the authors advance algorithms to coordinate a formation of mobile agents when the agents can only measure the ranges to their immediate neighbors. This solution requires that subsets of non-neighboring agents localize the relative positions of their neighbors while these are stationary, and then move to minimize a cost function.

There are also numerous strategies designed with marine vehicles in mind. A solution that decouples the controllers for formation shape, formation motion and vehicle orientation, but requires position information is proposed in [11]. Coordinated path following approaches are presented in [12] and [13], the latter specifically dealing with underwater pipeline inspection. These assume that the path to be followed is known to all vehicles, and generally work by exchanging some along-path synchronization measure.

An example real-world AUV operation making use of formation control is documented in [14].

\section{B. Problem statement}

The control problem discussed in this paper is illustrated in Fig. 1, which shows two leading vehicles (vehicles 2 and 3, represented as $x_{2}$ and $x_{3}$ ), moving along a certain unknown path, and a follower (vehicle 1 , represented as $x$ ), which we control. Through the remainder of this paper, and unless otherwise noted, the absence of an index indicates a variable or parameter related to vehicle 1 , the controlled or trailing vehicle.
The objective is for the trailing vehicle to follow the leaders in a triangular formation, at the same desired distance $d$ from each. In the figure, $x$ should converge to the desired position $x_{d}$. There exists a symmetric solution to the problem, with the desired position $x_{d}$ mirrored in relation to the segment defined by $x_{2} x_{3}$. We designate the solution shown in Fig. 1 by following motion, and the mirrored solution by leading motion. In the remainder of this paper, we will only deal with the case of following motion.

Let $z_{i}=\left\|x_{i}-x\right\| ; i=2,3$ denote the distances from the trailing vehicle to each of the leaders. From the range measurements, we define the common and differential mode errors

$$
\begin{aligned}
& \epsilon=\frac{e_{2}+e_{3}}{2}=\frac{z_{2}+z_{3}}{2}-d \\
& \delta=e_{3}-e_{2}=z_{3}-z_{2},
\end{aligned}
$$

respectively with $e_{i}=z_{i}-d ; i=2,3$. The control problem consist of deriving control laws to drive $\epsilon$ and $\delta$ to zero or, equivalently, to drive $x$ to $x_{d}$.

\section{RANGE-ONLY FORMATION CONTROL}

We first review the strategy designed to regulate the motion of the controlled (trailing) vehicle using only range information. As stated previously, we assume that the vehicle starts from a following position, in order to converge to a following motion. We further assume that the leader vehicles (2 and 3) move at a distance $d$ from each other, according to simple kinematics described by

$$
\dot{x}_{i}=\left[\begin{array}{c}
v_{i} \cos \psi_{i} \\
v_{i} \sin \psi_{i}
\end{array}\right], \quad i=2,3
$$

where $\left(v_{2}+v_{3}\right) / 2=v_{f}$ is the formation speed. The control signals are the linear velocity $v$ and the heading $\psi$, and the kinematic model of the follower is given by

$$
\dot{x}=\left[\begin{array}{l}
v \cos \psi \\
v \sin \psi
\end{array}\right],
$$

where $x \in R^{2}$ denotes its Cartesian position. Here, we assume that both leaders move with a common heading $\psi_{f}=\psi_{2}=\psi_{3}$, and that the total velocity vector of each leading vehicle is always perpendicular to the line segment that joins them. The heading $\psi_{f}$ is unknown to vehicle 1 .

Separate controllers are designed to stabilize each error measure, with the speed controller stabilizing the common mode error and the heading controller stabilizing the differential mode error. What follows is a brief review of the resulting controllers; intermediate steps in the derivation and proofs of convergence are omitted for brevity, but can be found in [2].

\section{A. Error dynamics}

From the definition of $z_{i}$, it follows that

$$
\dot{z}_{i}=v_{i} \cos \left(\alpha_{i}-\psi_{f}\right)-v \cos \left(\alpha_{i}-\psi\right)
$$


Simple computations show that the relations between $\alpha_{i}$ and the interior angles of the triangle $\theta_{i}$ in Fig. 1 are given by

$$
\begin{aligned}
& \alpha_{2}=\theta_{2}+\psi_{f}-\frac{\pi}{2} \\
& \alpha_{3}=-\theta_{3}+\psi_{f}+\frac{\pi}{2} .
\end{aligned}
$$

The law of cosines yields the following expressions for $\theta_{i}$ :

$$
\begin{aligned}
& \theta_{2}=\arccos \left(\frac{z_{2}^{2}+d^{2}-z_{3}^{2}}{2 d z_{2}}\right) \\
& \theta_{3}=\arccos \left(\frac{z_{3}^{2}+d^{2}-z_{2}^{2}}{2 d z_{3}}\right) .
\end{aligned}
$$

Although the control strategy can be applied to other types of trajectories, the next sections assume the simpler case of straight line constant-speed motion for the two leading vehicles. This means that $v_{2}=v_{3}=v_{f}$ and the simplified error dynamics for $\epsilon$ and $\delta$ become

$$
\begin{gathered}
\dot{\epsilon}=\cos \beta\left(v_{f} \cos \varphi-v \cos (\varphi+\tilde{\psi})\right) \\
\dot{\delta}=2 \sin \beta\left(v_{f} \sin \varphi-v \sin (\varphi+\tilde{\psi})\right),
\end{gathered}
$$

where

$$
\begin{aligned}
& \beta=\frac{\theta_{2}+\theta_{3}}{2}-\frac{\pi}{2} \\
& \varphi=\frac{\theta_{2}-\theta_{3}}{2},
\end{aligned}
$$

and $\tilde{\psi}=\psi_{f}-\psi$ is the heading error.

\section{B. Speed controller}

We propose the following speed controller to regulate the common mode error $\epsilon$ to zero:

$$
v=K_{p}^{s} \epsilon+K_{i} \int_{0}^{t} \epsilon d \tau
$$

where $K_{p}^{s}>0$ and $K_{i}>0$ are the proportional and integral gains, respectively. The rationale behind the proposed control law is that when the leader vehicles follow a straight-line trajectory with constant speed $v_{f}, \psi=\psi_{f}$ and $\delta=0$ (i.e., $x$ is on the perpendicular bisector of the $x_{2} x_{3}$ line segment), the dynamics of $\epsilon$ in (1) reduce to

$$
\dot{\epsilon}=\cos \beta\left(v_{f}-v\right),
$$

and, since $\cos \beta>0$, a control law $v=v_{f}+K_{p}^{s} \epsilon, K_{p}^{s}>0$ stabilizes exponentially the origin $\epsilon=0$, provided $\beta$ does not converge to $-\frac{\pi}{2}$. As $v_{f}$ is unknown, we include an integral term to adjust to it.

\section{Heading controller}

For the heading controller we propose the following control law that uses the differential mode error $\delta$ :

$$
\psi=\hat{\psi}_{f}+\gamma\left(K_{p}^{h} \delta\right),
$$

where $K_{p}^{h}>0, \hat{\psi}_{f}$ denotes an estimate of the formation heading $\psi_{f}$, and $\gamma$ is any function such that $\sin (\gamma($ ay $)) y>$ $0, \forall a>0$. An example is the saturation function $\gamma(y)=$ $\frac{\pi}{2} \operatorname{sat}(y)$.

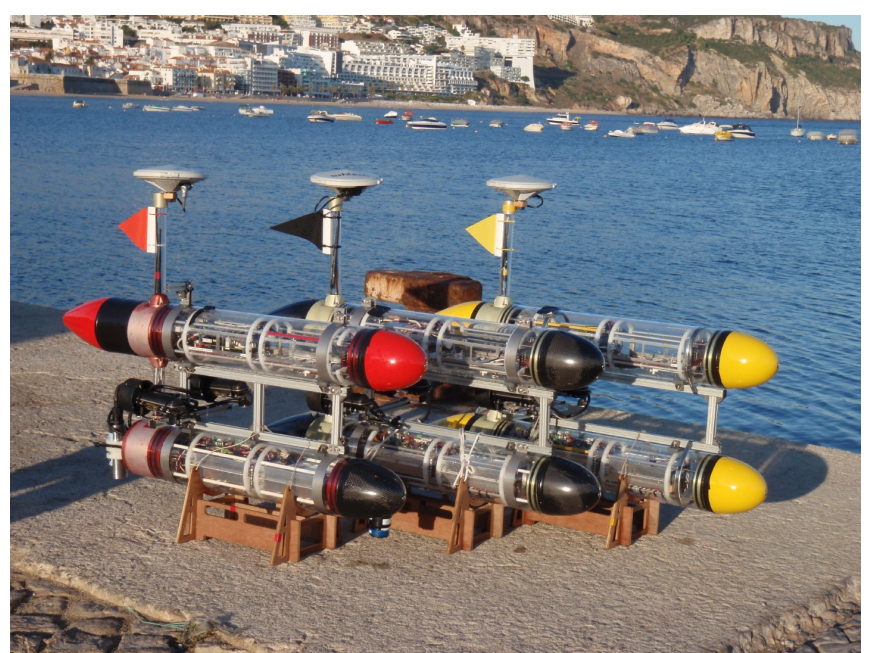

Fig. 2. The three MEDUSA AMVs being readied for deployment at an experimental site.

\section{Formation heading estimation}

In order to remove the dependence from the formation heading in (2), we can estimate this heading from the observed distances. Either of the following estimates for $\psi_{f}$

$$
\begin{aligned}
& \hat{\psi}_{f}^{2}=\arcsin \left(\frac{\xi}{v} \sin \left(\theta_{2}\right)-\frac{\dot{\hat{z}}_{2}}{v}\right)-\theta_{2}+\psi \\
& \hat{\psi}_{f}^{3}=\arcsin \left(-\frac{\xi}{v} \sin \left(\theta_{3}\right)+\frac{\dot{\hat{z}}_{3}}{v}\right)+\theta_{3}+\psi
\end{aligned}
$$

or their circular mean can be used. The exact values of $\dot{z}_{i}$ are not known, but can be estimated from the evolution of $z_{i}$ using a Kalman filter.

\section{ACOUSTICS-BASED FORMATION CONTROL FOR AUVS}

\section{A. Vehicle details}

The experimental evaluation of the algorithms for formation control was done using three MEDUSA-class autonomous semi-submersible robotic vehicles, developed at the Laboratory of Robotics and Systems in Engineering and Science (LARSyS), Instituto Superior Técnico, and shown in Fig. 2. Although the MEDUSAs are, for the time being, surface vehicles (a diving-capable version is currently under development), they are used here in place of AUVs. This has some practical advantages, as we can mimic the most relevant characteristics of an AUV while retaining a surface communication channel and GPS localization, respectively used for remote monitoring and ground truth.

Each MEDUSA-class vehicle weighs approximately $30 \mathrm{Kg}$ and consists of two longitudinal acrylic housings with a total length of around $1 \mathrm{~m}$. The upper body is partially above the surface and carries an EPIC single-board computer, an RTK-enabled GPS receiver, a full navigation sensor suite and an underwater camera. Most of the lower body is taken up by the batteries. An 802.11 interface is used for surface communications, while a Tritech acoustic modem enables 
underwater communication. The vehicle is propelled by two side-mounted, forward-facing stern thrusters that directly control surge and yaw motion, and is capable of speeds up to $1.5 \mathrm{~m} / \mathrm{s}$.

As the vehicle is bound to the horizontal plane, its kinematic equations take the simpler form

$$
\begin{aligned}
& \dot{x}=u \cos \psi-v \sin \psi \\
& \dot{y}=u \sin \psi+v \cos \psi \\
& \dot{\psi}=r,
\end{aligned}
$$

where $u$ (surge speed) and $v$ (sway speed) are the body axis components of the velocity of the vehicle, $x$ and $y$ are the Cartesian coordinates of its center of mass, $\psi$ defines its orientation (heading angle), and $r$ its angular velocity. The motions in heave, roll and pitch can be neglected, as the vehicle has large enough meta-centric height. The resulting simplified dynamic equations of motion for surge, sway and yaw are

$$
\begin{aligned}
m_{u} \dot{u}-m_{v} v r+d_{u} u & =\tau_{u} \\
m_{v} \dot{v}+m_{u} u r+d_{v} v & =0 \\
m_{r} \dot{r}-m_{u v} u v+d_{r} r & =\tau_{r},
\end{aligned}
$$

where $\tau_{u}$ stands for the external force in surge (thruster common mode), $\tau_{r}$ for the external torque (thruster differential mode), and the terms

$$
\begin{aligned}
m_{u} & =m-X_{\dot{u}} & & d_{u}=-X_{u}-X_{|u| u}|u| \\
m_{v} & =m-Y_{\dot{v}} & & d_{v}=-Y_{v}-Y_{|v| v \mid}|v| \\
m_{r} & =I_{z}-N_{\dot{r}} & & d_{r}=-N_{r}-N_{|r| r|r|}|r| \\
m_{u v} & =m_{u}-m_{v} & &
\end{aligned}
$$

represent vehicle masses and hydrodynamic added masses, and linear and quadratic hydrodynamic damping effects. The full set of MEDUSA physical parameters can be found in [15].

\section{B. Implementation constraints}

While the controllers developed show good performance under the assumptions made during their derivation, moving to a real-world implementation requires some significant changes.

First and foremost, ranges in an underwater setting are most often measured using acoustic equipment, by registering the time of flight of an echo request and reply. In our case, the ranging is done by the general purpose Tritech acoustic modem that equips the MEDUSA. The low transmission speed makes it so that we can only issue one echo request every few seconds. Since transmissions cannot overlap on the single common channel, time multiplexing must be used to obtain the ranges to each of the leader vehicles. We choose to query each one separately, although other solutions are possible, e.g., emitting a broadcast ping with vehicle-dependent delayed replies. Since both leaders have to be queried, a complete information update only occurs every 4 seconds. This is in stark contrast with our previous assumption of continuous measurement. To prevent changes to the algorithms, we have chosen to implement two hybrid Kalman filters that take the discrete samples and output a continuous estimate of the distances. The filters implement a simple model

$$
\begin{aligned}
& \dot{\zeta}_{1}=\zeta_{2} \\
& \dot{\zeta}_{2}=w \\
& z_{k}=\zeta_{1 k}+n_{k}
\end{aligned}
$$

where $w$ and $n_{k}$ respectively denote process and measurement noise. While a more accurate internal model of the inter-vehicle range evolution could yield better results, to do so would require extra information that is currently unknown. The range information received is never current, and comes with a latency of approximately 0.5 seconds, imposed by transmission times and I/O scheduling on both the sender and receiver. We decided not to implement any mitigation techniques (e.g., back-dating the filter updates), instead retaining the simplicity of the solution.

The measurements taken are inherently noisy. This noise is, for practical purposes, quite low - we did not fully characterize it, but the individual ranging error was predominantly under $0.5 \mathrm{~m}$ - but it again must be taken into account. The same way, outliers are inevitable, albeit infrequent. These are mostly caused by floor geometry and nonuniform propagation in the water, leading to the reception of an echo reply through a path other than the shortest one and resulting in an overestimation of the distance. We implemented a simple outlier filter based on a sliding window. Losses are also an inescapable reality, and need to be tolerated within reasonable limits.

Last, we come to the issue of formation heading determination. The originally proposed approach was designed for continuous measurements, and works well under that assumption. However, it depends on the first derivative of the measured ranges, and the low sampling rate on a real vehicle makes an estimate of this derivative very unreliable; for this reason, the approach used to estimate heading is not practical. However, the need for formation heading estimation is itself debatable: seeing as the vehicles use full-featured acoustic modems to measure ranges, it is possible to piggyback data on the ranging reply. This feature must be used with caution, in order not to over-extend communication times (thereby decreasing the sampling rate even further), but adding a single integer to the reply is without major consequences. Heading is the hardest parameter to estimate, and, unlike velocity, is widely available in most surface and underwater vehicles through the use of a relatively low-cost magnetic compass, a basic navigation aid. Transmitting the current heading (compressed to 1 byte) in the echo reply thus becomes an obvious choice.

In order to estimate the heading from the data, we feed another hybrid Kalman filter with the incoming values whenever a new range is received. Thus, while each range estimator is, in the absence of losses, updated every 4 seconds, the heading estimator is updated every 2 seconds. As the heading of both vehicles should be close to and converge to the formation heading, this allows for a higher quality and more responsive estimate. We have observed that 
transmitting the desired formation heading (identical in both vehicles) instead of the current heading (different in each vehicle) slightly improves the estimate, but decided against it as it requires an assumption on the information available to the leaders, sacrificing the generality of the solution.

An overview of the resulting implementation is presented in Fig. 3. Note that the expressions for the speed and heading controller in our implemented solution remain the same as previously presented: all changes take place in the earlier (leftmost) stages. Also note that while we are using a surface vehicle, the solution is applicable for constant-depth underwater operations and, with minor changes, to variabledepth underwater vehicles equipped with a depth sensor and independent depth control.

\section{EXPERIMENTAL EVALUATION}

\section{A. Simulation results}

The expected performance of the algorithms was first assessed in simulation, prior to deployment and testing in the water. Simulations were run using a full dynamic model of the MEDUSA. This model has been used for simulations and hardware-in-the-loop testing, and has been fitted with extensive experimental data, providing an accurate representation of the real dynamics of the vehicle.

Range measurements are extracted directly from the position difference, to which uncorrelated zero-mean Gaussian noise $(\sigma=0.2)$ was added. Ranges to each vehicle are obtained with a 4-second period, meaning there is a new sample from one of them every 2 seconds. Our simulation does not consider packet loss: although its effect can be potentially devastating, previous real-world experiments in our scenario had shown it to be low to moderate.

For the sequence presented here, the leader vehicles are configured to navigate the first 3 legs of a standard lawnmower path at an average speed $v_{f}=0.5 \mathrm{~m} / \mathrm{s}$, well within the capabilities of the MEDUSA. The vehicles are expected to form an equilateral triangle of side $d=13 \mathrm{~m}$. The resulting path can be seen in Fig. 4.

Figures 5 and 6 show some metrics related to the performance of the algorithm. The common mode error $(\epsilon)$ and the speed $(v)$ remain fairly stable and close to the reference values after a brief initial transient. The differential mode error shows some variation, although generally under $4 \mathrm{~m}$.

Looking at the recorded headings, one can also see an apparent delay in responding to the beginning of a turn: this is not the case. While there is a certainly a delay imposed by the Kalman filter (and the overall response time of the system), the controlled vehicle is in fact trailing the leaders by a few seconds; in order to remain in formation, it needs to slightly delay the start of the turn.

\section{B. Real-world testing}

Real world trials were conducted in June 2012 at Parque das Nações in Lisbon, Portugal. This is a fairly sheltered saltwater bay connected to the Tagus estuary. It provides for ample space for testing, with minimal currents and good conditions for assembly of the base station. Water depth is however limited (generally under $5 \mathrm{~m}$ ), which limits

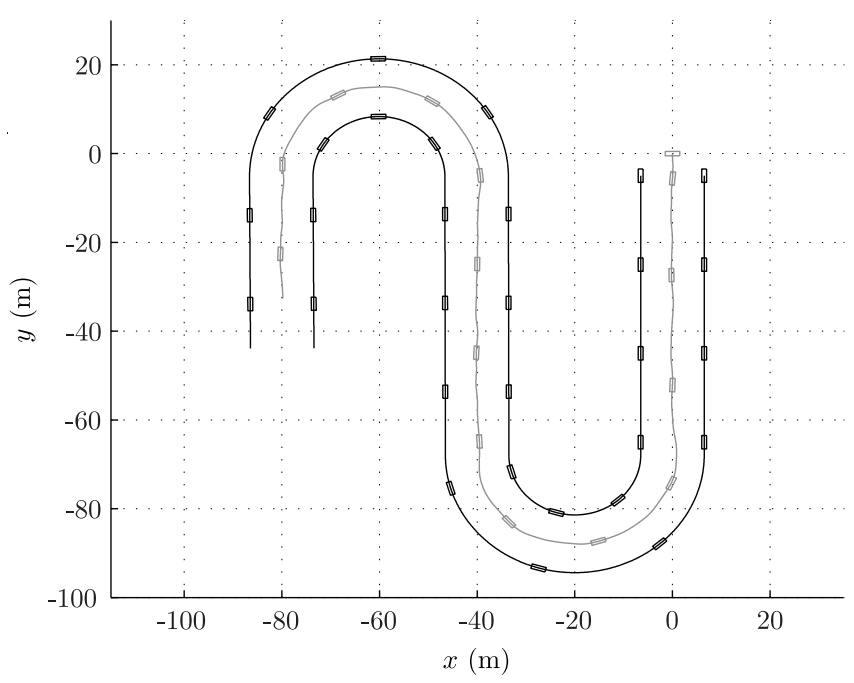

Fig. 4. Path described by the three vehicles during the simulation run. The starting position is in the upper right corner, and the follower is in the middle, in light gray.
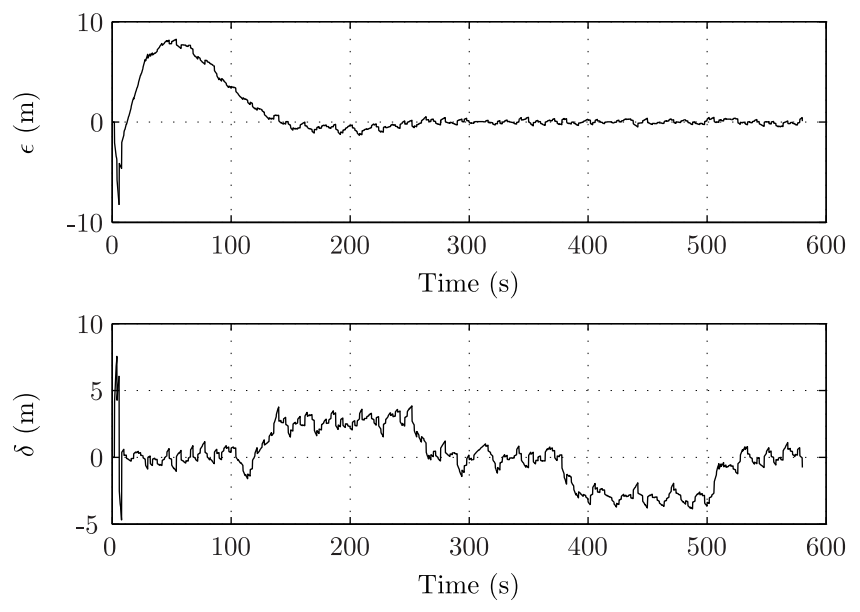

Fig. 5. Common and differential mode errors in a simulation of the lawnmower maneuver shown above.

the performance of the acoustic communication systems. The settings for the trials follow the constraints previously discussed, as well as the approximate parameters selected for the simulation. The vehicles were set to a triangular formation with $d=13 \mathrm{~m}$, with the leaders navigating at an approximate speed of $v_{f}=0.4 \mathrm{~m} / \mathrm{s}$.

The vehicles execute a series of increasing complexity validation and evaluation tests, culminating in the 3-legged lawnmower maneuver presented below, spanning around $120 \mathrm{~m} \times 120 \mathrm{~m}$. They were equipped with the full sensor suite, including RTK GPS, but the trailing vehicle only logs the data for ground truth and does not use it, in any way, for navigation. The leader vehicles are running the Coordinated Path Following algorithm described in [15]. A short video of the experiment can be found in the accompanying material.

Figure 7 shows a top view of the paths described by the three vehicles, starting in the upper right corner. A transient can be noticed at the beginning: none of the vehicles start in 


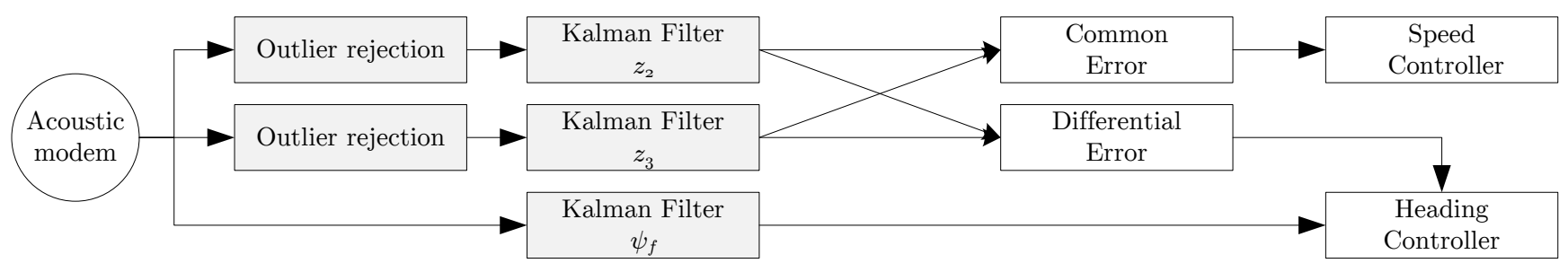

Fig. 3. Structure and data flow in the MEDUSA implementation of the formation control algorithm. Modules in gray perform the conversion of available discrete data to the continuous signals required the algorithm. The remaining modules are the same as in the previous discussion.
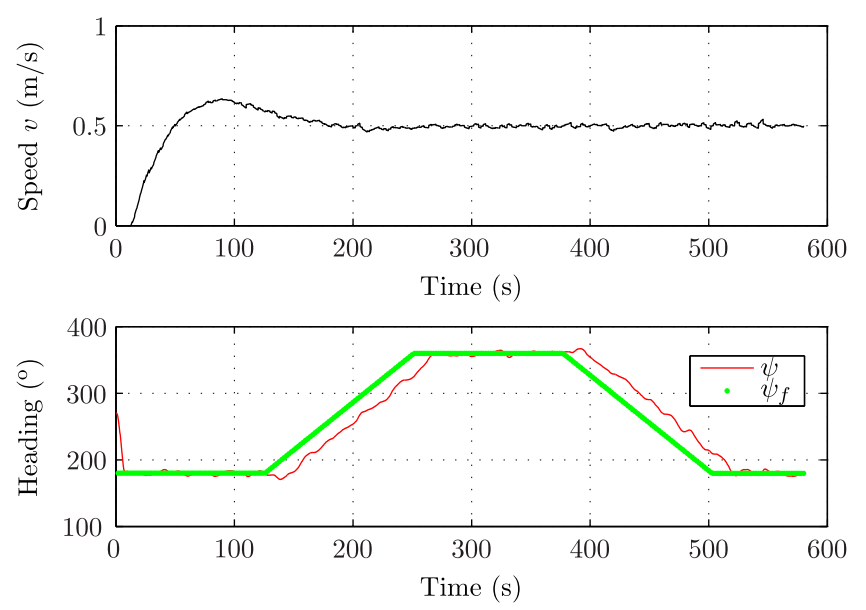

Fig. 6. Speed and heading of the follower vehicle in a simulation of a lawnmower maneuver. The green dots represent $\psi$ samples received from the leaders.

their designated position or heading and need to adjust. The movement of the leader and their rapidly varying reported headings impact the Kalman filter estimate, causing the controlled vehicle to start in a non-ideal direction.

Minimal packet loss was observed during the trials. When it takes place, it is mostly at the start of turns, due to misalignment of the acoustic modems in the three vehicles, and it is the leading factor causing the vehicle to stray off path. In spite of this, Fig. 8 shows that the errors maintain a similar curve to the one obtained in simulation, with the exception of isolated spikes in the common mode error. A look at Fig. 9 confirms these two phenomena. While $\psi_{2}$, $\psi_{3}$ and, consequently, the $\psi_{f}$ samples almost overlap during the majority of the test, there are significant deviations in the first 40 seconds. Some missing samples are visible at the start and end of turns, for instance around the $150 \mathrm{~s}$ and 290 s marks.

Finally, Fig. 10 shows a comparison of the real distances measured using GPS data with the estimates produced by the Kalman filters used to transform the discrete acoustic range measurements into a continuous signal. Despite the clearly visible sawtooth pattern, typical of hybrid filters, it is generally able to closely follow the real distance.

\section{CONCLUSions AND OUTLOOK}

This paper discussed a solution to a three-vehicle formation keeping problem where a follower moves in a triangular

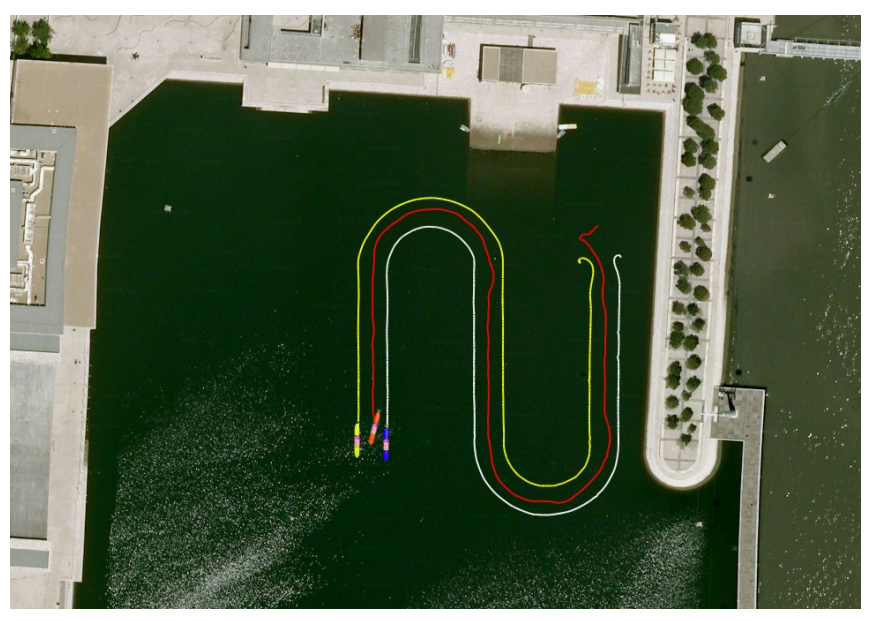

Fig. 7. Aerial view of the mission area and trajectory (real data). The maneuver starts on the top right corner, and the trajectory of the controlled vehicle is shown in red.

formation behind two leading vehicles, using inter-vehicle range measurements with no a priori knowledge of the path taken by the leaders. An algorithm using only range information was first reviewed, followed by a more realistic solution tied to a real vehicle, that considers a discrete and noisy measurement model with low sampling rate and uses additional heading information piggybacked on the acoustic echo reply.

The proposed solution was implemented and tested on the MEDUSA vehicles. The evaluation results show good performance, with minimal disturbance under straight lines, even in the presence of packet loss, sensor noise and outliers. The hybrid Kalman filters used are able to accurately estimate the distances, despite the low rate of the acoustic ranges, and the piggybacked heading information allows for a smoother response to changes in direction. Furthermore, the results obtained are comparable to those predicted using simulation.

Future work will address the implementation and testing of the algorithm on a underwater setting, using the new submersible MEDUSA vehicle in development at IST. This will explicitly introduce a third dimension to the problem, but we do not foresee a significant impact. Extending the algorithm to a higher vehicle count, while working on the same basic model, is also in our plans, as is the pairing of the algorithm with robust methods for initialization and collision avoidance. Although not explored here, offline merging of 

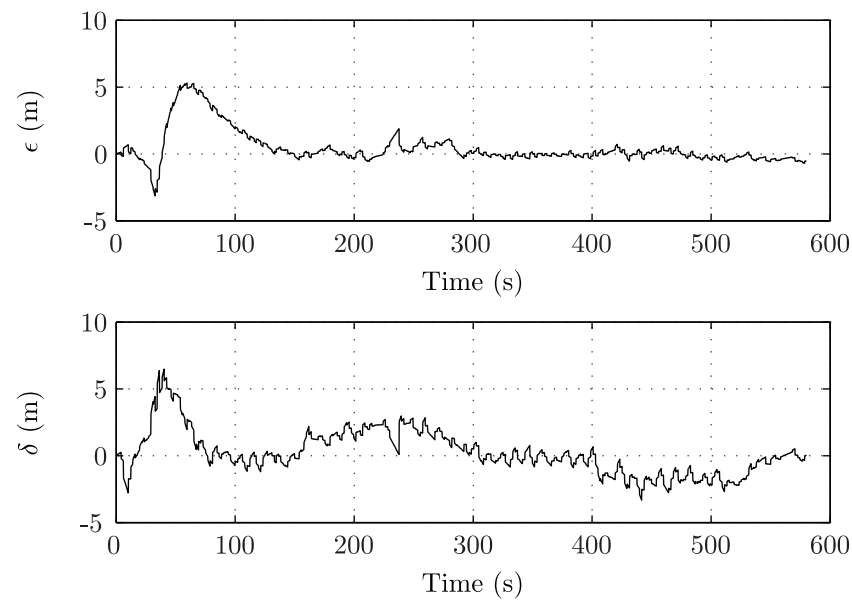

Fig. 8. Time evolution of the common mode and differential mode errors along the lawnmower path.
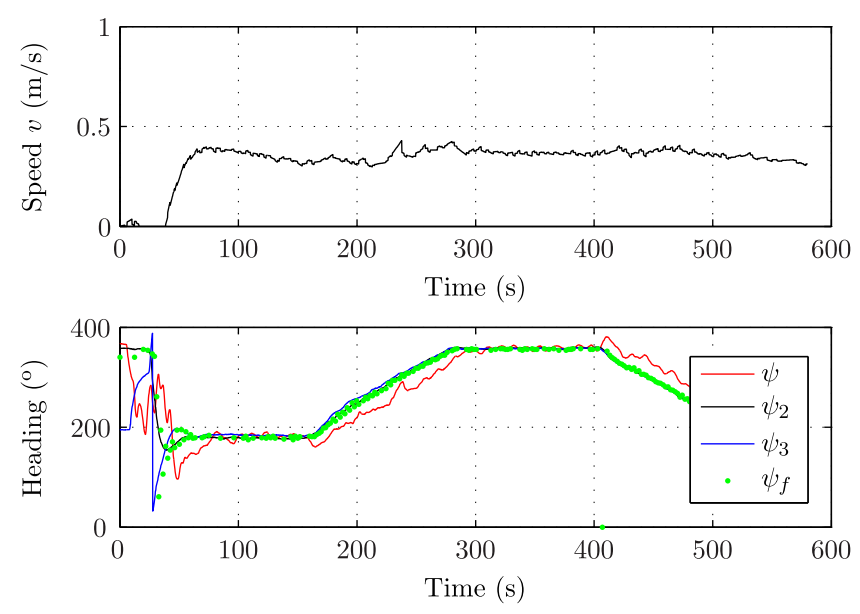

Fig. 9. Time evolution of the follower vehicle speed $v$, and headings $\psi, \psi_{2}$, $\psi_{3}$, as well as the references received acoustically, $\psi_{f}$. The vehicle speed is estimated from GPS measurements, and has non-negligible associated noise.

the leader and follower logs will make it possible to reconstruct the AUV path and geolocate any measurements taken. Finally, open sea trials will allow us to test and validate the algorithm with stronger currents, waves and winds. Further testing and development of the algorithm is currently taking place in the context of the EU FP7 project MORPH.

\section{ACKNOWLEDGMENTS}

We gratefully acknowledge the DSOR team for their efforts in the development of the MEDUSA vehicles and their support and collaboration on the planning and execution of our sea trials, especially J. Botelho, J. Ribeiro, L. Sebastião, M. Rufino, M. Bayat, P. Góis and V. Hassani. We also acknowledge M. Gallieri for his early work on this subject.

\section{REFERENCES}

[1] T. Lochmatter, E. Göl, I. Navarro, and A. Martinoli, "A Plume Tracking Algorithm based on Crosswind Formations," in Proc. of the Tenth Int. Symp. on Distributed Autonomous Robotic Systems. Lausanne, Switzerland: Springer Tracts in Advanced Robotics (2013), Vol. 83, pp. 91-102., 2010.
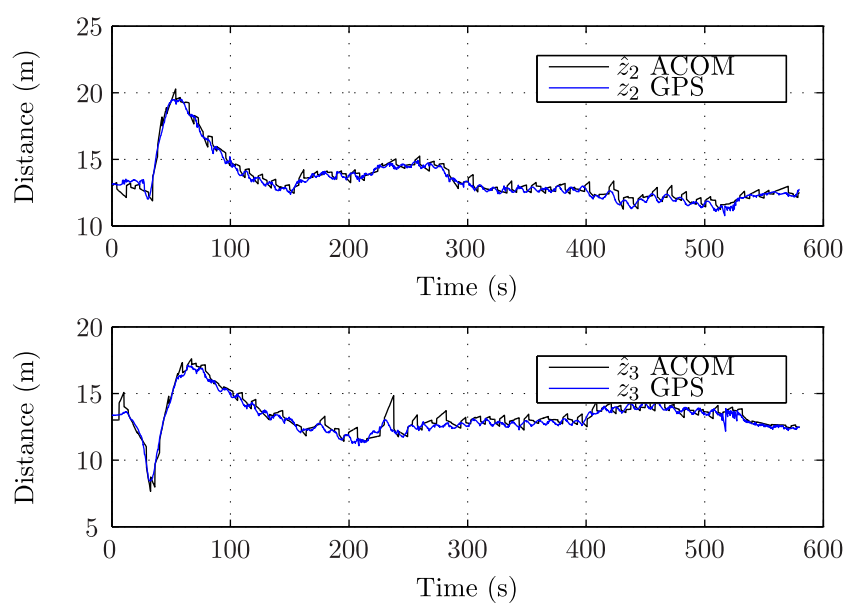

Fig. 10. Time evolution of the distances $z_{i}$ measured using GPS data and their estimates $\hat{z}_{i}$ obtained from the acoustic ranges by a Kalman filter.

[2] J. M. Soares, A. P. Aguiar, and A. M. Pascoal, “Triangular formation control using range measurements : an application to marine robotic vehicles," in IFAC Workshop on Navigation, Guidance and Control of Underwater Vehicles (NGCUV2012), Porto, Portugal, 2012.

[3] J. Desai, J. Ostrowski, and V. Kumar, "Modeling and control of formations of nonholonomic mobile robots," Robotics and Automation, IEEE Transactions on, vol. 17, no. 6, pp. 905-908, 2001.

[4] R. Falconi, S. Gowal, and A. Martinoli, "Graph-based distributed control of non-holonomic vehicles endowed with local positioning information engaged in escorting missions," in 2010 IEEE International Conference on Robotics and Automation. Anchorage, Alaska, USA: IEEE, May 2010, pp. 3207-3214.

[5] M. Cao and A. S. Morse, "Station keeping in the plane with rangeonly measurements," in 2007 American Control Conference. New York, NY, USA: IEEE, Jul. 2007, pp. 5419-5424.

[6] K.-K. Oh and H.-S. Ahn, "Formation control of mobile agents based on inter-agent distance dynamics," Automatica, vol. 47, no. 10, pp. 2306-2312, Oct. 2011.

[7] A. N. Bishop, "Distributed bearing-only formation control with four agents and a weak control law," in 2011 9th IEEE International Conference on Control and Automation (ICCA). Santiago, Chile: IEEE, Dec. 2011, pp. 30-35.

[8] — , "A Very Relaxed Control Law for Bearing-Only Triangular Formation Control," in Proceedings of the 18th IFAC World Congress, Milano, Italy, Aug. 2011, pp. 5991-5998.

[9] M. Basiri, A. N. Bishop, and P. Jensfelt, "Distributed control of triangular formations with angle-only constraints," Systems \& Control Letters, vol. 59, no. 2, pp. 147-154, Feb. 2010.

[10] M. Cao, C. Yu, and B. D. O. Anderson, "Formation control using range-only measurements," Automatica, vol. 47, no. 4, pp. 776-781, Apr. 2011.

[11] H. Yang and F. Zhang, "Geometric formation control for autonomous underwater vehicles," in 2010 IEEE International Conference on Robotics and Automation. Anchorage, Alaska, USA: IEEE, May 2010, pp. 4288-4293.

[12] R. Ghabcheloo, A. P. Aguiar, A. M. Pascoal, C. Silvestre, I. Kaminer, and J. Hespanha, "Coordinated path-following in the presence of communication losses and time delays," SIAM Journal on Control and Optimization, vol. 48, no. 1, p. 234, 2009.

[13] X. Xiang, B. Jouvencel, and O. Parodi, "Coordinated Formation Control of Multiple Autonomous Underwater Vehicles for Pipeline Inspection," International Journal of Advanced Robotic Systems, vol. 7, no. 1, p. 1, 2010.

[14] N. E. Leonard, D. A. Paley, F. Lekien, R. Sepulchre, D. M. Fratantoni, and R. E. Davis, "Collective Motion, Sensor Networks, and Ocean Sampling," Proceedings of the IEEE, vol. 95, no. 1, pp. 48-74, Jan. 2007.

[15] J. Ribeiro, "Motion Control of Single and Multiple Autonomous Marine Vehicles," Master's Thesis, Instituto Superior Técnico - Technical University of Lisbon, 2011. 